\title{
Multi-Agent System Architecture for Zero Defect Multi-stage Manufacturing
}

\author{
Paulo Leitão ${ }^{1,2}$, José Barbosa ${ }^{1,3}$, Carla A. S. Geraldes ${ }^{1}$, João P. Coelho ${ }^{1,3}$ \\ 1 Polytechnic Institute of Bragança, Campus Sta Apolónia, 5300-253 Bragança, Portugal, \\ Email: \{jbarbosa, pleitao, carlag, jpcoelho\} @ipb.pt \\ 2 LIACC - Artificial Intelligence and Computer Science Laboratory, Rua Campo Alegre 1021, \\ 4169-007 Porto, Portugal \\ 3 INESC-TEC, Rua Dr. Roberto Frias, 4200-465 Porto, Portugal
}

\begin{abstract}
Multi-stage manufacturing, typical in important industrial sectors, is inherently a complex process. The application of the zero defect manufacturing (ZDM) philosophy, together with recent technological advances in cyber-physical systems (CPS), presents significant challenges and opportunities for the implementation of new methodologies towards the continuous system improvement. This paper introduces the main principles of a multi-agent CPS aiming the application of ZDM in multi-stage production systems, which is being developed under the EU H2020 GO0D MAN project. In particular, this paper describes the MAS architecture that allows the distributed data collection and the balancing of the data analysis for monitoring and adaptation among cloud and edge layers, to enable the earlier detection of process and product variability, and the generation of new optimized knowledge by correlating the aggregated data.
\end{abstract}

Keywords: Cyber-physical systems, Zero defect manufacturing, Multi-agent systems, Multi-stage manufacturing.

\section{Introduction}

Nowadays, the modern and competitive companies must be able to track rapid technological changes while carrying out the manufacture of products with complex features, which commonly requires the assembly of a large number of components. Moreover, the dynamic nature of today's manufacturing environments, compels organizations to an incessant reassessment in an effort to respond to continuous challenges in the field of manufacturing management.

Multi-stage manufacturing systems [7], which are typical in important industrial sectors, such as automotive, house hold appliance and semiconductor manufacturing, are inherently complex. Among others characteristics, it is common to have multiple stages with mixed sequential and/or parallel configurations, feedback/feedforward loops, and mixed data types that arise from multiples processes. In this context, the application of the zero defect manufacturing (ZDM) philosophy [10], together with recent technological advances in cyber-physical systems (CPS), namely Internet of Things (IoT), big data, and advanced data analytics, presents significant challenges and opportunities to develop new methodologies aiming the continuous improvement of process efficiency and product quality. 
In the actual European manufacturing context, the development and transfer of advanced technology for the industry is of strategic importance. This technological contribution aims to boost competitiveness while targeting several aspects of the manufacture processes such as reduce waste and costs, and increase processes efficiency and quality tracking. In this context, the EU H2020 GO0D MAN (aGent Oriented Zero Defect Multi-stage mANufacturing) project (see http://go0dman-project.eu/) aims to integrate and combine process and quality control for multi-stage manufacturing systems using a distributed system architecture built upon an agent-based CPS and smart inspection tools designed to support ZDM strategies.

In this approach, the multi-agent system (MAS) [11] infra-structure, combined with data analytics, provides real-time and early identification of deviations allowing to prevent the occurrence of defects at a single stage and their propagation to downstream processes, enabling the global system to be predictive, by early detecting faults, and proactive, by self-adapting to different conditions. This approach is aligned with Industry 4.0 trends, contributing for achieving a dynamic and continuous system improvement in multi-stage manufacturing environments, addressed by the ZDM philosophy.

The objective of this paper is to introduce the main principles of the designed agentbased CPS to support zero defects multi-stage manufacturing. The proposed architecture is based on the previous successful EU FP7 GRACE project [5], adapted to handle the idiosyncrasies introduced by multi-stage manufacturing systems and ZDM strategies, providing an infra-structure for the distributed intelligence and digitization.

The rest of the paper is organized as follows: Section 2 overviews the challenges of applying ZDM in multi-stage manufacturing systems and Section 3 describes the agentbased CPS architecture to implement ZDM strategies. Section 4 describes the distribution of data analysis by cloud and edge layers and Section 5 presents some interaction patterns to implement the ZDM strategies. Finally, Section 6 rounds up the paper with the fundamental concluding remarks and points out some directions for future work.

\section{Challenges of Applying ZDM in Multi-stage Manufacturing}

The ZDM philosophy aims to achieve a steady-state production with no defects whatsoever at the end of the manufacturing process. This concept encompasses a large number of mechanisms that can be used to guarantee and maintain a manufacturing system without defects. If a company is capable to achieve a production plan with (near) zerodefects, then an increase in the profits and customers loyalty is expected. The former is accomplished by eliminating the costs associated to scrap production and rework and the latter by providing to the customers products with the required characteristics.

At the present time, the ZDM paradigm is an open question to debate since, as usually described, attaining a zero-defect production seems an unrealistic task. However, ZDM must be analysed within distinct frames of reference according to the type of manufacturing process. In a single stage manufacturing, the ZDM paradigm must be observed at steady-state production. The methodology must be able to promote adaptability and fast recover in the presence of disturbances such as machine malfunction or degradation and differences in the incoming raw material. In multi-stage production, the attaining of ZDM is even more complex since it is necessary to track and foresee even- 
tual small disturbances in each station that can lead, at the final production process, to a large deviation in the product quality standards. This performance improvement can be done by continuously monitor the relevant state variables scattered along the production stages and through proactive methods and computational intelligence algorithms, to reduce quality variability by anticipative reaction to possible defects. Usually, continuous improvement is made by using a repeated and cyclical questioning based on two well adopted models: PDCA (Plan-Do-Check-Act) cycle and DMAIC (Define-MeasuresAnalyse-Improvement-Control) Six-Sigma cycle [3]. By applying continuously these cycles, improvement becomes part of all performed activities [9].

The majority of the existing methodologies towards ZDM are concentrated on the quality of final product instead of considering all the existing activities and/or stages within the manufacturing system. Also, to achieve ZDM in a multi-stage manufacturing system, it is mandatory to consider all the complex data relationships within a specific stage and between different stages. Having this in mind, the requirements for implementing ZDM strategies in multi-stage environments are the following:

- Distributed continuous collection of data coming from operators, equipment, in course products or final products for each stage.

- Continuous data analysis within the stage and among stages to support the real time monitoring to evaluate equipment, product and process, as well as the real-time adaptation of process and inspection parameters aiming a system improvement.

- Continuous global data analysis to support knowledge generation aiming optimisation, root cause analysis, fault diagnosis and/or fault prognosis.

The adoption of these requirements may support better decisions allowing to detect and prevent the occurrence of failures and reducing waste reaching ZDM performance and improving the productivity of a company. Multi-stage manufacturing is characterized by its distributed nature, which makes even more difficult the efficient operation of traditional rigid and monolithic control structures, since they are not, any longer, able to respond and adapt close to real time and efficiently to condition changes or process deviations. In a ZDM strategy context, the use of a MAS CPS infra-structure completely matches this distributed nature, and supports the early detection of anomalies and properly implement mitigation actions for preventing the defect generation and propagation to downstream stages and to reduce waste.

These dynamic monitoring mechanisms for the real-time and early detection of events should be complemented with global data analytics and simulation aiming to extract new knowledge and optimisation strategies supporting the analysis and control steps of the DMAIC cycle.

\section{Architectural Design Principles for the Multi-agent CPS}

Aiming to face the identified challenges towards ZDM strategies in multi-stage manufacturing, a distributed and intelligent infra-structure becomes mandatory to collect, monitor and process data according to the different industrial requirements, namely in terms of responsiveness and optimisation. For this purpose, the GOODMAN CPS architecture integrates production and quality control, in multi-stage manufacturing at different ISA 95 levels, by using MAS principles. 


\subsection{Multi-agent Systems to Implement the Distributed Intelligence}

MAS play a key role to implement a distributed CPS that should be able to collect, monitor and process data, in a distributed manner, at local and global levels, supporting the fast detection of process and product variability, the implementation of preventive or corrective actions, and the prediction of process faults or performance degradation, allowing to design optimisation strategies for the continuous system improvement. MAS will also contribute to reach a more modular, scalable, flexible and robust system, being the plugability and reconfigurability also simplified and executed on-the-fly.

In the proposed work, the MAS CPS is based on the GRACE MAS architecture [5], and comprises a society of distributed, autonomous and cooperative intelligent agents representing the production components disposed along the multi-stage manufacturing system (see Fig. 1). In some cases, these agents have a direct association to a physical resource, e.g., a welding robot or a quality control station, but in other cases can represent logical entities, such as data analytics tools.

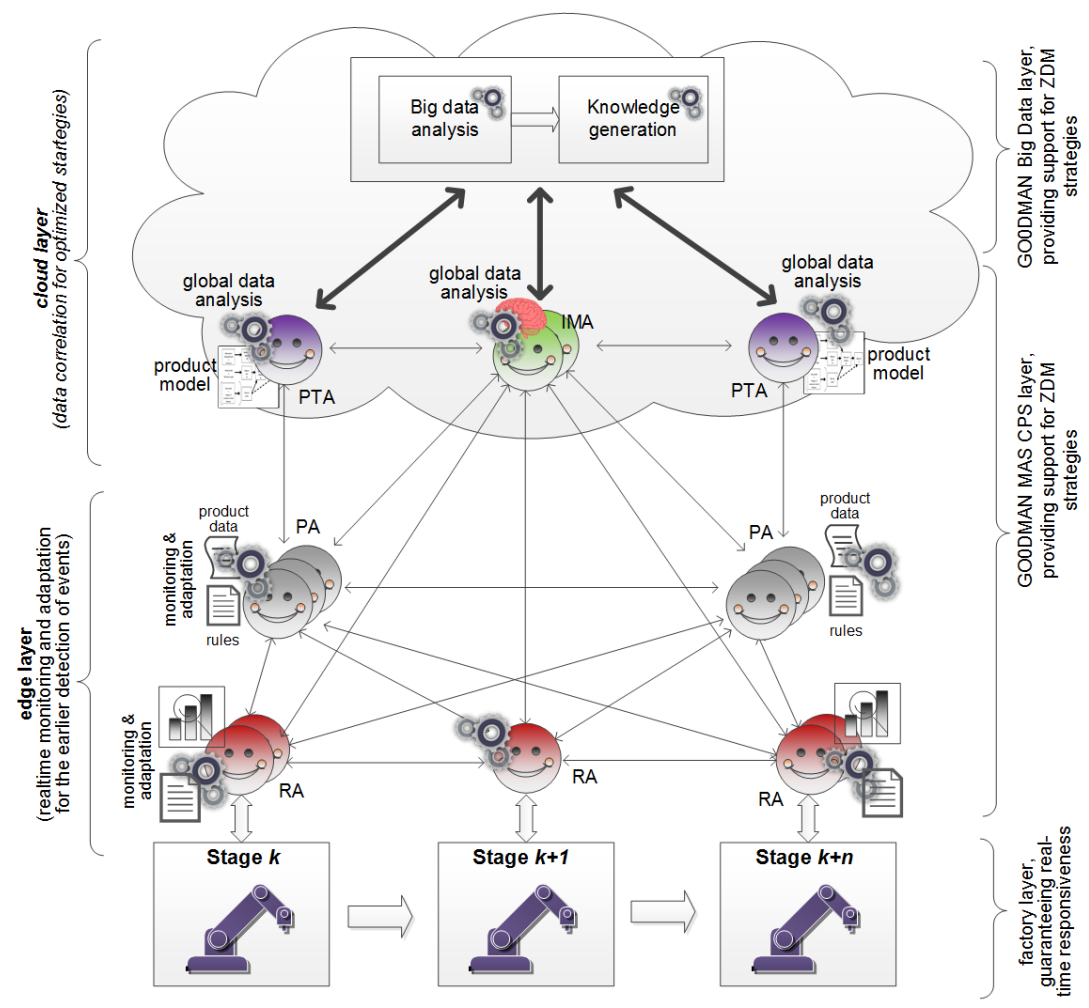

Fig. 1: GOODMAN MAS CPS architecture for multi-stage manufacturing.

These software agents interacting with physical processes are able to collect data in distributed manner. Even the products being produced along the line have associated an 
intelligent agent, which transforms a passive product into an intelligent one [4], with capabilities to gather and store data from its historical production process and current operation, supporting the real-time monitoring and decision-making.

The agents are enriched with data analytics enabling the rapid reaction to critical situations, and particularly the earlier identification of anomalies, deviations and patterns leading to condition changes or defects, and the application of proper mitigation strategies for self-adaptation and optimisation according to several criteria (e.g., adjustment of the operations' parameters). Agents placed at the higher decision level can interact with big data analytic and knowledge generation tools to get insight information related to the system optimisation based on the overall data correlation.

These agents are able to interact among each other according to some coordination patterns aiming to share their knowledge and capabilities, and consequently capable to implement inter-stage coordination policies towards the ZDM strategies. Particularly, the implementation of feedback control loops for preventing the occurrence and/or propagation of defects is achieved by the interaction among the agents.

\subsection{Agents: Roles and Functionalities}

The designed MAS CPS distributes the intelligence by four types of agents, identified according to the particularities of the production system: Product Type Agents (PTA), Product Agents (PA), Resource Agents (RA) and Independent Meta Agents (IMA). The existing relationships among these agents, and also their main functions, are illustrated in the UML Class diagram represented in Fig. 2.

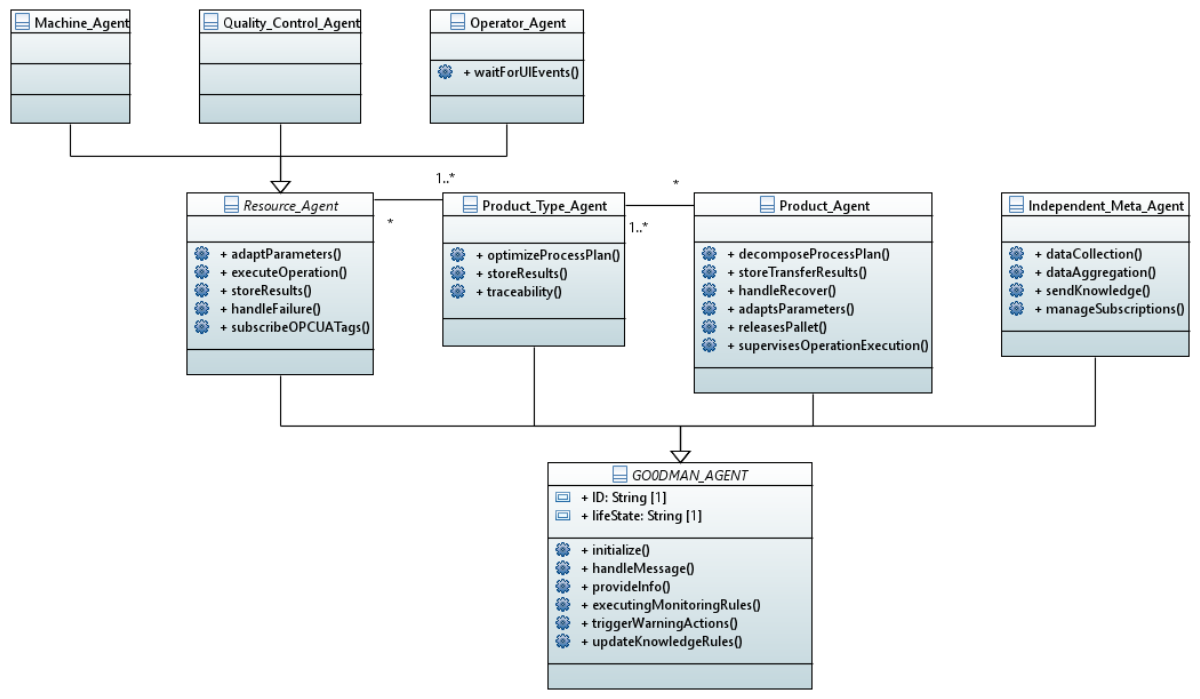

Fig. 2: UML class diagram for the GOODMAN agent types. 
PTAs represent the catalogue of products that can be produced in the production line and contains the process and product knowledge required to produce the product, namely the process plan. These agents, 1 agent for each product model, are responsible for the following main functions:

- Launching PAs according to the production orders.

- Collection and storage of data related to the execution of the products.

- Monitoring the on-going production of products related to a specific model.

- Optimisation and adjustment of the process plan associated to the product model.

- Traceability of the produced products in the production line.

PAs manage the production of product instances in the production line (e.g., an oven or a car) according to a process plan that specifies how to produce the product. They ( 1 agent for each product being produced, and in case of batch production, 1 agent for each batch) are responsible for the following main functions:

- Collection and storage of production data related to the execution of the product.

- Adaptation of the process and inspection parameters of operations to be executed by the resources, according to the local knowledge and historical data.

- Monitoring the evolution of the product production along the line to detect, amongst others, possible deviations from the plan and quality degradation.

- Pre-processing of the collected data to filter those that are sent to IMAs (mitigating the latency and bandwidth).

RAs are associated to physical resources disposed along the production line, such as robots, inspection stations and operators, and are responsible to manage the execution of their operations. Several specializations of this agent class can be considered, e.g., Machine Agents (MA), Quality Control Agents (QCA) and Operator Agents (OA). These agents, 1 for each resource, are responsible, among others, for the:

- Collection and storage of data related to the process stations, using proper IoT technologies, e.g., OPC-UA (Open Platform Communications - Unified Architecture). In case of OAs, the integration uses a proper HMI (Human-Machine Interface).

- Adaptation of the operations' parameters, according to the local knowledge and historical data from the resource perspective.

- Monitoring the evolution of the resource performance to detect, amongst others, possible quality of service degradation.

- Pre-processing of the collected data to filter those that are sent to IMAs (mitigating the latency and bandwidth problems).

The PA and RA agents uses knowledge, e.g. based on a set of rules, to monitor the process evolution and adapt the operation parameters, which is periodically adjusted by IMAs that use the results from data analysis and knowledge generation running at the cloud level. In fact, IMAs act at a strategic level to provide global system optimisation by managing the data aggregation and generation of new knowledge, as follows:

- Aggregation of the data collected in a distributed way from the system's agents.

- Management of the subscription of optimisation services requested by local agents. 
- Monitoring the system performance to detect improvement opportunities.

- Triggering the batch data analysis aiming to identify meaningful correlations and new knowledge towards the optimisation of the production system.

- Propagation of new or adjusted knowledge to the local agents.

Since IMA agents are positioned at a strategic level and have larger response time, these agents work in a strong collaboration with big data analysis and knowledge generation tools that will run powerful data analytics algorithms considering larger datasets to achieve better optimisation and data correlation.

These agents can be classified according to several criteria regarding the decisionmaking, namely the responsiveness, optimisation, data volume, data heterogeneity and reasoning, as illustrated in Fig. 3.

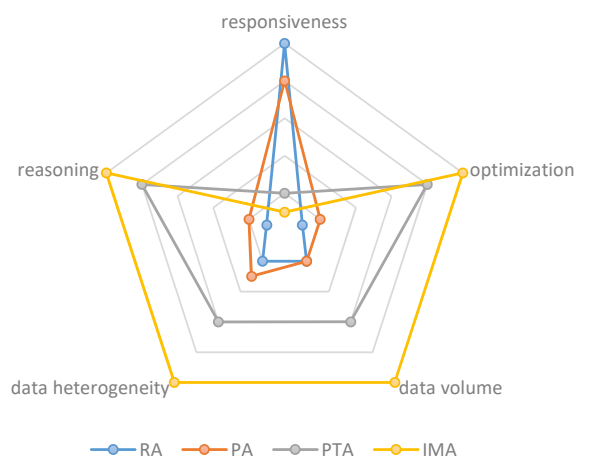

Fig. 3: Classification of the different types of GOODMAN agents.

As clearly showed, RAs and PAs focus on responsiveness by using simple reasoning algorithms to handling low data volume, while PTAs and IMAs focus on optimisation by using powerful data analytics algorithms to handle huge amounts of data without critical time restrictions.

\section{Data Analysis at Edge and Cloud Levels}

Data analysis algorithms, knowledge management approaches and learning techniques are applied in the proposed MAS CPS to enhance the implementation of the ZDM strategies in multi-stage manufacturing environments. Taking advantage of the MAS capabilities, the data analytics is performed in a distributed manner and spread by different levels, namely at cloud and edge levels, according to the expected temporal response and degree of optimisation, as illustrated in Fig. 4.

Local agents, i.e. RAs and PAs, operate at edge and run faster response data analysis algorithms for the knowledge extraction to allow the automatic real-time monitoring, detecting discrepancies between desired and actual process values, and the fast selfdiagnosis, self-adaptation and predictive maintenance. As example, these agents are 


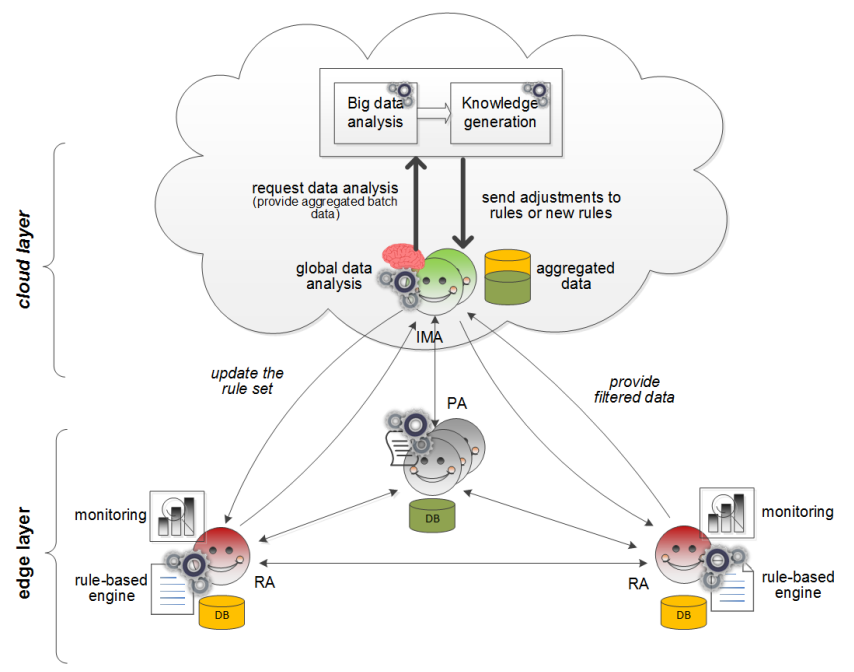

Fig. 4: Adjustment/generation of rules at cloud level and execution of rules at edge level.

running a set of rules, previously defined by the cloud level, included in their knowledge base for the early detection of deviations and trends.

These rules can be atomic or composed. The atomic rules considers the variability of only one parameter, e.g., based on the well-known decision rules defined by [2] and posteriorly extended by [6] to enhance the sensitivity of control charts allowing the detection of process changes, for example:

Rule 1: IF "One point falls outside the three-sigma control limits" THEN "trigger action Type 1"

Rule 2: IF "Two out of three consecutive data points fall beyond the two-sigma warning limits" THEN "trigger action Type 2"

Rule 3: IF "Six points in a row steadily increasing or decreasing" THEN "trigger action Type 3"

Normally, the identification of the product/process variability occurs if any one or more rules from the rules-set are met. The composed rules consider the variability of more than one parameter, which dependency is identified by the big data analysis tool, and posterior knowledge generated by the knowledge management running in the cloud layer. An example of such kind of rule is the following:

Rule 4: IF "One point from parameter A falls outside the three-sigma control limits" AND "Four out of five consecutive points from parameter B fall beyond the one-sigma limit" THEN "trigger action Type 4"

The different parameters considered in the composed rules can come from the same stage or from different stages. In this last case, the RA needs to interact with other RAs to get information that will be used in the rule execution. However, PAs can handle the execution of composed rules comprising data from different stages in an easy manner 
since they have collected data related to the execution of their products (and e.g., can use it to adapt the set of functional tests to be executed at the end of the production line).

These agents are also able to supply the on-line gathered information to meta agents placed at the higher level, i.e. PTAs and IMAs, which aggregates and performs data fusion. The latest, operating at the cloud level, are running more powerful data analytics algorithms to get richer conclusions from aggregated data and allowing to generate new knowledge, e.g., adjusting the existing rules or creating new ones, that is used to optimise the predictive task of early detecting defects and preventing their propagation to downstream stages. The agents at this level provides self-optimisation and continuous improvement using also cloud based infra-structures for data storage and processing. Note that at this stage, the response time is not critical, being possible to run algorithms to deeply mine the data to find useful knowledge and correlations.

This distribution of data collection and analysis among different layers is strongly advised in the emergent industrial cyber-physical systems, where we "should not send all collected data to be processed in the cloud but instead to make analysis in the edge" as sustained by James Truchard from National Instruments at IFAC IMS'16, and "enable the feedback of intelligence through the system to update control for optimal production", sustained by Brian Weiss from NIST in the same event. Thus, the establishment of local and global levels for the data collection and analysis is the core architectural axiom by implementing ZDM strategies for the multi-stage manufacturing environment, since it allows to combine the earlier detection of anomalies or process/product variability locally at each stage, with the possibility to aggregate and correlate data from different individual stages aiming to identify anomalies that can only be detected globally. The challenge is the proper balancing of the local data analysis - providing automatic and real-time monitoring and early detection of deviations and trends and the global data analysis - providing self-optimisation and continuous improvement.

\section{Interaction Patterns Towards ZDM Strategies}

The global behaviour of the multi-agent system emerges from the interaction among the distributed agents, following proper interaction patterns to achieve ZDM strategies. This section illustrates these patterns by describing the interaction pattern to support the adaptation of the rules running locally based on the generation of new knowledge and the interaction pattern to support the early detection of events.

Fig. 5 depicts the message sequence diagram that supports the dynamic adaptation of rules that is based on the two-fold trigger process. First, the agents that run at the edge nodes, i.e. RAs and PAs, are continuously sending data to IMAs. All the agents are proceeding with respective ETL (Extract, Transform and Load) techniques allowing to exchange data in a efficient manner. The store of data at the IMA level (i.e. the load operation from ETL) allows for the big data tool to obtain operative data for longterm and optimized processing. After processing the data, and in the case where new, possible, patterns are detected, the big data tool feeds the results to the knowledge management tool which responsibility is the generation of knowledge to the data, e.g., generating or updating rules. At this stage, the knowledge management tool informs the 
IMA of the changes, which on turn analyses them, decomposing the receiver agents, and informing them accordingly.

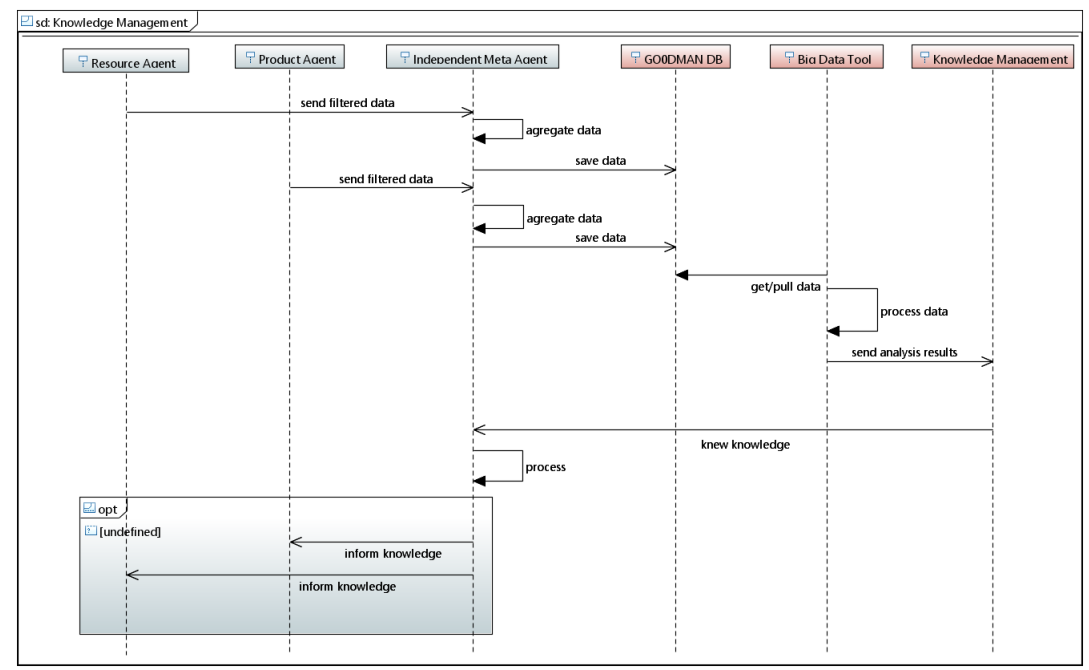

Fig. 5: Interaction among agents for the adaptation of rules based on the knowledge generation.

Additionally, the need for a rule update could come directly from a trigger of the PAs or RAs. These agents, by being continuously managing their execution, are able to detect unknown patterns and behaviours, that could need further, higher level, analysis or even that rules might be tighten, improving the productive process and consequently increase their quality (by decreasing the variability possibilities). In fact, when such situation appears, the designated agent requests a rule update check to the IMA, that requests such verification to the big data tool by invoking it and by providing identified data. After this step, the process is similar of the one previously described, culminating in an update or new generation of rules to the designated agent.

A second example, illustrated in Fig. 6, is related to the interaction between agents for the early detection of events considering rules that use parameters from different stages. As previously described, PAs are responsible to manage its own execution. One of its main features is the decomposition of the process plan and, for each step, the PA adapts the parameters for the next execution, considering the previous execution parameters. Therefore, the PA, before issuing a processing initialization sends the operation parameters to the RA.

After executing the operation, the RA analyses the operation results and sends them to the PA. At this stage, both the PA and the RA consider this new output into their life-cycle aiming at different perspectives. The RA considers this to properly analyse its own process execution, enabling a continuous process optimization and to detect future problematic situations at the physical resource level. Additionally, the RA cooperate with upstream and downstream RAs, advising them to proceed with corrective 


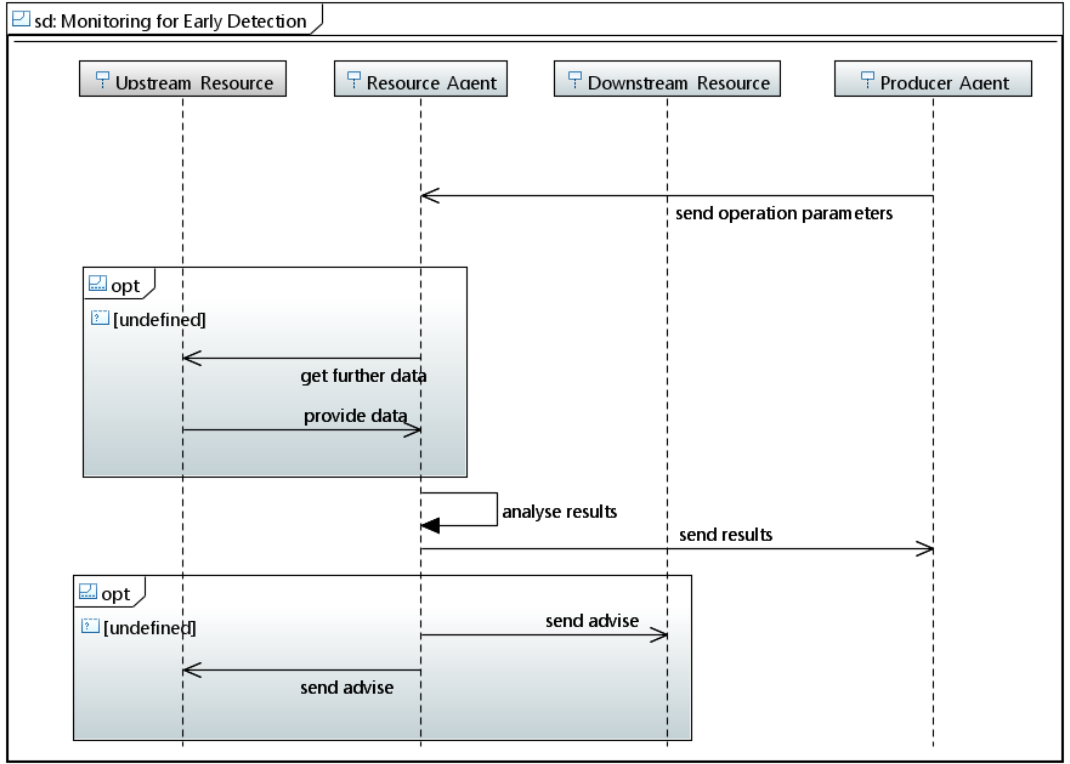

Fig. 6: Interaction among agents for the earlier detection of defects downstream propagated.

measures enabling the whole process quality standards to be meet, although its execution quality is being degraded. On the other side, the continuous monitoring of the PA enables to forecast and predict future product quality issues, allowing a parameter adjustment for the subsequent RAs, mitigating problematic situations, while maintains and increases the product quality. As example, PAs can simply adjust the parameters of the next operation or the set of texts to be executed in the inspection station.

\section{Conclusion}

Quality management can be considered a key concern of almost manufacturing organizations since high-quality products can give an organization a significant competitive advantage. Good quality products reduces the costs of rework, waste, complains and returns and, most important, generates satisfied customers. The majority of the existing quality control and improvement methodologies for multi-stage manufacturing systems were built upon quantitative modelling of the system (see [8] for more details). Due to its inherent complexity, this type of manufacturing system presents significant challenges and opportunities, at both operational and tactical levels, aiming to achieve a (near) zero defect manufacturing. One of the challenges is to continuously collect, monitor and analyse huge amount of data within a single stage and among different stages to periodically generate knowledge. The generated knowledge will support better decisions allowing to detect and prevent the occurrence of failures reaching ZDM performance and improving the productivity of a company. 
In this context, this paper introduces the main principles of a multi-agent CPS aiming the application of ZDM in multi-stage manufacturing systems. This concept is being developed under the EU H2020 GO0D MAN project. Along this article, the MAS architecture was introduced by analysing the roles and functions of each agent, the way these agents are distributed along two layers, the roles of data analysis in each layer, and finally the way the overall system emerges from the interaction among individual agents. At this point, two examples of interaction patterns towards the implementation of the ZDM strategies were illustrated, namely the interaction among agents placed at the edge level for the early detection of defects, and the interaction between agents place in cloud and edge levels aiming the knowledge generation.

Future work is related to the further specification of the multi-agent CPS architecture concerning the interfaces with data analytics tools and physical process and inspection equipment and legacy systems, and the implementation of the designed solution by using a proper multi-agent development framework, e.g., the Java Agent Development Framework (JADE) platform [1].

\section{Acknowledgement}

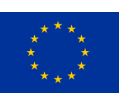

This work his part of the GO0D MAN project that has received funding from the European Union's Horizon 2020 research and innovation programme under grant agreement $\mathrm{N}^{\circ} 723764$.

\section{References}

1. Bellifemine, F., Caire, G., Greenwood, D.: Developing Multi-Agent Systems with JADE. Wiley (2007)

2. Electric, W.: Statistical Quality Control Handbook. Western Electric Corporation (1956)

3. Gryna, F., Chua, R., DeFeo, J.: Juran's Quality Planning and Analysis, 5th ed. McGraw-Hill (2006)

4. Leitão, P., Rodrigues, N., Barbosa, J., Turrin, C., Pagani, A.: Intelligent products: The GRACE experience. Control Engineering Practice 42, 95-105 (2015)

5. Leitão, P., Rodrigues, N., Turrin, C., Pagani, A.: Multi-agent System Integrating Process and Quality Control in a Factory Producing Laundry Washing Machines. IEEE Transactions on Industrial Informatics 11(4), 879-886 (2015)

6. Montgomery, D.C.: Introduction to Statistical Quality Control, 6th ed. John Wiley \& Sons, Inc. (2009)

7. Shi, J.: Stream of Variation Modeling and Analysis for Multistage Manufacturing Processes. CRC Press (2007)

8. Shi, J., Zhou, S.: Quality control and improvement for multistage systems: A survey. IEEE Transactions pp. 744-753 (2009)

9. Slack, N., Brandon-Jones, A., Johnston, R.: Operations Management, 7th ed. Pearson (2013)

10. Wang, K.S.: Towards zero-defect manufacturing $(\mathrm{zdm})-$ a data mining approach. Advances in Manufacturing 1(1), 62-74 (2013)

11. Wooldridge, M.: An Introduction to Multi-Agent Systems. John Wiley and Sons (2002) 